\title{
The Role of Collagen Triple Helix Repeat-Containing 1 Protein (CTHRC1) in Rheumatoid Arthritis
}

\author{
Askhat Myngbay ${ }^{1}$, Limara Manarbek ${ }^{2}$, Steve Ludbrook ${ }^{3}$ and Jeannette Kunz ${ }^{2, *}$ \\ 1 PhD Program in Science Engineering and Technology, Nazarbayev University; \\ askhat.myngbay@nu.edu.kz \\ 2 Department of Biology, School of Sciences and Humanities, Nazarbayev University, Nur Sultan, \\ Kazakhstan; 1manarbek@nu.edu.kz \\ 3 GlaxoSmithKline Research \& Development, Stevenage, United Kingdom; Steve.B.Ludbrook@gsk.com \\ * Correspondence: jeannette.kunz@nu.edu.kz; Tel.: +7-702-411-0463
}

Received: date; Accepted: date; Published: date

\begin{abstract}
Rheumatoid arthritis (RA) is a chronic autoimmune disease, causing inflammation of joints, cartilage destruction and bone erosion. Biomarkers and new drug targets are actively sought and progressed to improve available options for patient treatment. The Collagen Triple Helix Repeat Containing 1 protein (CTHRC1) may have an important role as a biomarker for rheumatoid arthritis, as CTHRC1 protein concentration is significantly elevated in the peripheral blood of rheumatoid arthritis patients, compared to osteoarthritis (OA) patients and healthy individuals. CTHRC1 is a secreted glycoprotein that promotes cell migration and has been implicated in arterial tissue-repair processes. Furthermore, high CTHRC1 expression is observed in many types of cancer and this is associated with cancer metastasis to the bone and poor patient prognosis. However, the function of CTHRC1 in RA is still largely undefined. The aim of this review is to summarize recent findings on the role of CTHRC1 as a potential biomarker and pathogenic driver of RA progression. We will discuss emerging evidence linking CTHRC1 to the pathogenic behavior of fibroblast-like synoviocytes and to cartilage and bone erosion through modulation of the balance between bone resorption and repair.
\end{abstract}

Keywords: Collagen triple helix repeat containing 1; CTHRC1; rheumatoid arthritis; biomarker; bone erosion; cartilage destruction; fibroblast-like synoviocytes; Wnt signaling

\section{Introduction}

Rheumatoid arthritis (RA) is a chronic systemic autoimmune disease, which affects around $1 \%$ of the population according to the World Health Organization [1]. RA is characterized by cartilage degradation and bone erosion within both small and larger joints including hand, wrist, knee and feet, leading to disability in a proportion of patients [2]. Although several genetic and environmental factors have been linked to an increased risk for RA, the definitive pathogenesis remains obscure, making the development of effective treatment strategies challenging. Some breakthroughs, including the introduction of anti-tumor necrosis factor alpha (anti-TNF $\alpha$ ) in the treatment of RA, which occurred in the mid-1990s, showed efficacy towards inflammation and joint destruction and led to an improvement of clinical outcomes of RA [3]. However, cytokine antagonists against TNF $\alpha$, Interleukin-6 (IL-6) and Interleukin-1 (IL-1) lacked efficacy in a significant fraction of patients and the persistence of efficacy remains a tremendous problem even in responsive patients [3]. Likewise, the therapeutic effects of $\mathrm{B}$ cell depletion and $\mathrm{T}$ cell co-stimulation blockers were not observed in all patients $[4,5]$. Indeed, accumulating evidence suggests that differences in genetic background and exposure to environmental stimuli among patients require treatment strategies to be more personalized as particular patients' response can be TNF $\alpha$-dominant, T cell-dominant, and B celldominant $[4,5]$. 
While significant efforts have been undertaken to identify biomarkers to diagnose RA, there is still a lack of diagnostic and prognostic biomarkers for better patient stratification. ACPA (anticitrullinated protein antibody) and RF (rheumatoid factor) are two widely accepted autoantibodies associated with progressive autoimmune disease and are used in clinical practice as biologic markers of RA [6]. Interestingly, ACPA and RF are detected in some patients' prior to the development of arthritis, providing an opportunity for early intervention [6]. Nonetheless, neither of these two markers has sufficient specificity or sensitivity for effective diagnosis of all RA patients and neither autoantibody allows classification of patient subpopulations or patient outcome [6]. Recently, CTHRC1 has emerged as a new biomarker that may contribute to improved RA diagnosis and assessment of disease activity. CTHRC1 protein levels are increased in the plasma of RA patients, but were either absent or detected only at very low levels in healthy individuals or patients suffering from other forms of arthritis, such as OA or reactive arthritis (ReA, [7]). These findings suggest that CTHRC1 may enhance differential diagnosis of RA when used in a wider panel of markers. In addition, emerging evidence suggests that CTHRC1 is directly involved in the disease course. Accordingly, CTHRC1 is expressed in a subset of activated fibroblast-like cells (RA-FLS) of the synovium associated with RA pathogenesis [8]. Furthermore, CTHRC1 is also secreted from osteocytes and/or osteoclasts and acts a critical modulator of bone resorption and formation $[9,10]$, thus raising the possibility that CTHRC1 levels may reflect a more direct role - pathological or protective - in cartilage and bone erosion in RA. In this review, we will discuss the role and diagnostic potential of CTHRC1 in RA and provide an overview of the signaling processes modulated by CTHRC1.

\section{Expression and function of CTHRC1 during tissue remodeling}

CTHRC1 is a secreted $30 \mathrm{kDa}$ glycoprotein that is highly conserved among vertebrates. The protein contains a N-terminal hydrophobic signal peptide that directs CTHRC1 for secretion, a short collagen triple helix repeat (CTHR) domain consisting of 12 repeats of the Gly-X-Y motif [11], and a conserved C-terminal domain with structural homology to the globular C1q domain of collagens VIII and X domain [12]. The CTHR domain may promote CTHRC1 dimer or trimer formation and mediate interaction with a variety of ligands $[11,12]$. Several shorter alternatively spliced transcripts have been described, one of which lacks the N-terminal hydrophobic signal peptide and may not be secreted. However, only the longest transcript has so far been functionally characterized in more detail.

CTHRC1 was first identified by Pyagay et al. who reported the transiently upregulated expression of CTHRC1 in rat arteries on adventitial and intimal smooth muscle following injury [11]. Increased levels of CTHRC1 protein were also observed in the matrix of calcifying human atherosclerotic plaques [9,10,13-16]. Notably, CTHRC1 was not detectable in normal arteries, indicating that the protein plays a specific role in the wound healing response and promotes vascular remodeling during arterial injury [11,17]. Mechanistically, increased CTHRC1 levels are associated with a significant decrease in collagen type I and type III mRNA and protein levels, leading to a reduction of collagen deposition, and enhanced migratory capability [11].

Consistent with such a role, CTHRC1 expression has been correlated with conditions associated with deregulated wound and tissue repair, including liver fibrosis [16,18-21] and liver injury caused by Hepatitis B infection [14,15,22]. Increasing evidence also links CTHRC1 to tumor progression: CTHRC1 overexpression promotes epithelial-mesenchymal transition and metastasis in various types of cancer and is associated with poor patient prognosis [23-27]. In addition, CTHRC1 was shown to promote tumor angiogenesis by stimulating endothelial cell proliferation, migration and capillary-like tube formation [28]. Exogenously added CTHRC1 induced the recruitment of Tie2expressing monocytes to the tumor microenvironment in vivo in mice by stimulating the expression of the Tie2 receptor ligand angiopoietin-2 (Ang-2) in endothelial cells leading to increased tumor neovascularization [28,29]. Conversely, treatment with a CTHRC1-neutralizing antibody abrogated Ang-2 expression and reduced tumor neovascularization and tumor burden [28].

Under non-pathological conditions, CTHRC1 expression is detected mainly during embryonic development in the visceral endoderm, the notochord and neural tube and is also significantly 
associated with developing cartilage and bone, especially in calcified tissues [30]. In the adult body, expression of CTHRC1 in the bone is more restricted and observed only in the bone matrix and periosteum, but not the articular cartilage [30]. CTHRC1 expression is further detected in the myocardium and renal artery and is increased specifically in stromal cell types, including myofibroblasts and smooth muscle cells [30,31]. In addition, CTHRC1 is expressed in human pituitary glands and was proposed to act as a circulatory hormone [32]. Overall, the restricted expression in the adult body likely accounts for the low levels of CTHRC1 protein normally detected in circulation.

\section{Signaling roles of CTHRC1}

\subsection{Role of CTHRC1 in the TGF $\beta$ pathway}

During vascular development and upon injury, CTHRC1 expression can be activated by transforming growth factor $\beta$ (TGF- $\beta$ ) signaling through SMAD proteins and by Bone morphogenetic protein 2 (BMP2) and 4 (BMP4) $[9,17,33]$. The promoter region of CTHRC1 has a consensus binding site for SMAD transcription factors (Figure 1), which are downstream components of TGF- $\beta /$ BMP4 pathways [34], thus providing a potential mechanism for the observed induction of CTHRC1 expression in response to activation of TGF- $\beta$ and BMP signaling pathways. TGF- $\beta$ plays a central role in the regulation of collagen synthesis via activation of SMAD2/3 complexes and mediates several negative regulatory effects during vessel repair leading to increased collagen deposition and smooth muscle cell proliferation that result in lumen narrowing [17]. Importantly, the induction of CTHRC1 expression by TGF- $\beta$ has an antagonistic effect on TGF- $\beta$ signalling with CTHRC 1 inhibiting the phosphorylation of SMAD2/3 in vascular cells (Figure 1, [33]). Elevated levels of CTHRC1 thereby promote vessel repair by inhibiting the expression of the TGF- $\beta$ target genes collagen type I and III, which leads to reduced collagen deposition and enhanced cell migration and vascular remodeling $[11,17,33]$. This regulatory feedback loop (summarized in Figure 1) may allow for tight control of CTHRC1 activity to balance the effects of TGF- $\beta$ during the wound repair process.

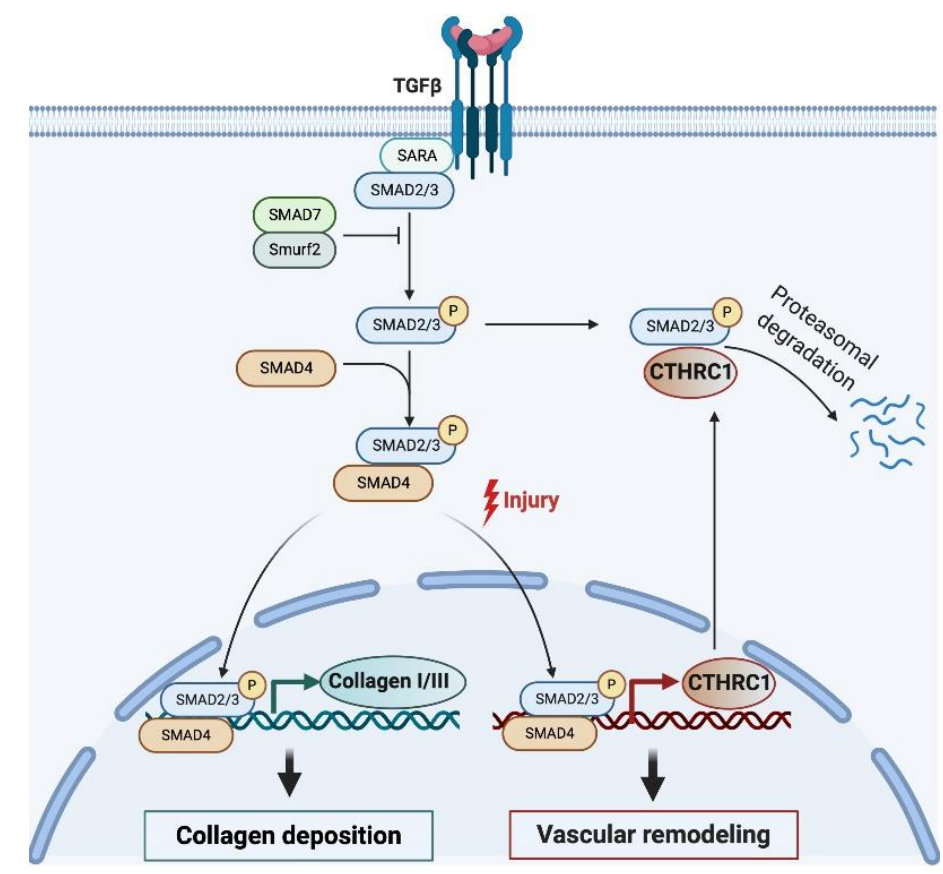

Figure 1. A model for CTHRC1 modulating collagen deposition and vascular repair following injury through the TGF- $\beta$ signaling pathway. Elevated levels of CTHRC1 eventually Wnt/Fzd/Ror2 lead to attenuation of TGF- $\beta$ signaling by inhibiting SMAD2/3 phosphorylation [33], possibly leading to SMAD2/3 degradation [16].

\subsection{CTHRC1 is a component of canonical and non-canonical Wnt signaling pathways}


CTHRC1 has also been linked to elements of the canonical and noncanonical branches of the Wnt (Wingless and Int-1) signaling pathway. Activation of the canonical branch of the pathway leads to the stabilization and nuclear translocation of the transcriptional activator $\beta$-catenin into the cell nucleus where $\beta$-catenin promotes the transcription of Wnt-associated genes [35]. Canonical Wnt signaling is implicated in embryonic development, cancer, and stem cell differentiation [35,36]. Besides the canonical branch, there are several non-canonical branches of the pathway, which do not lead to the cytoplasmic stabilization of $\beta$-catenin. One of these pathways is the planar cell polarity (PCP) pathway, which regulates cell motility and adhesion via Dvl (Dishevelled), RhoA, and actin cytoskeletal reorganization [35].

Prevailing evidence suggests that canonical and non-canonical Wnt pathways converge at the level of CTHRC1. N-glycosylation was reported to stabilize CTHRC1 protein and promote the secretion and pro-migratory function of CTHRC1 [37]. N-glycosylation appears to be mediated by the N-glycosidase DPAGT1, which is linked to Wnt/ $\beta$-catenin signaling [38]. Accordingly, DolichylPhosphate N-Acetylglucosaminephosphotransferase 1 (DPAGT1) and Wnt/ $\beta$-catenin synergize to upregulate CTHRC1 expression and protein levels ((Figure 2, [37]). CTHRC1 may also positively promote the transcriptional activity of either $\beta$-catenin or the $\beta$-catenin/TCF complex [37], although it remains to be delineated exactly how CTHRC1 regulates $W n t / \beta$-catenin signaling.

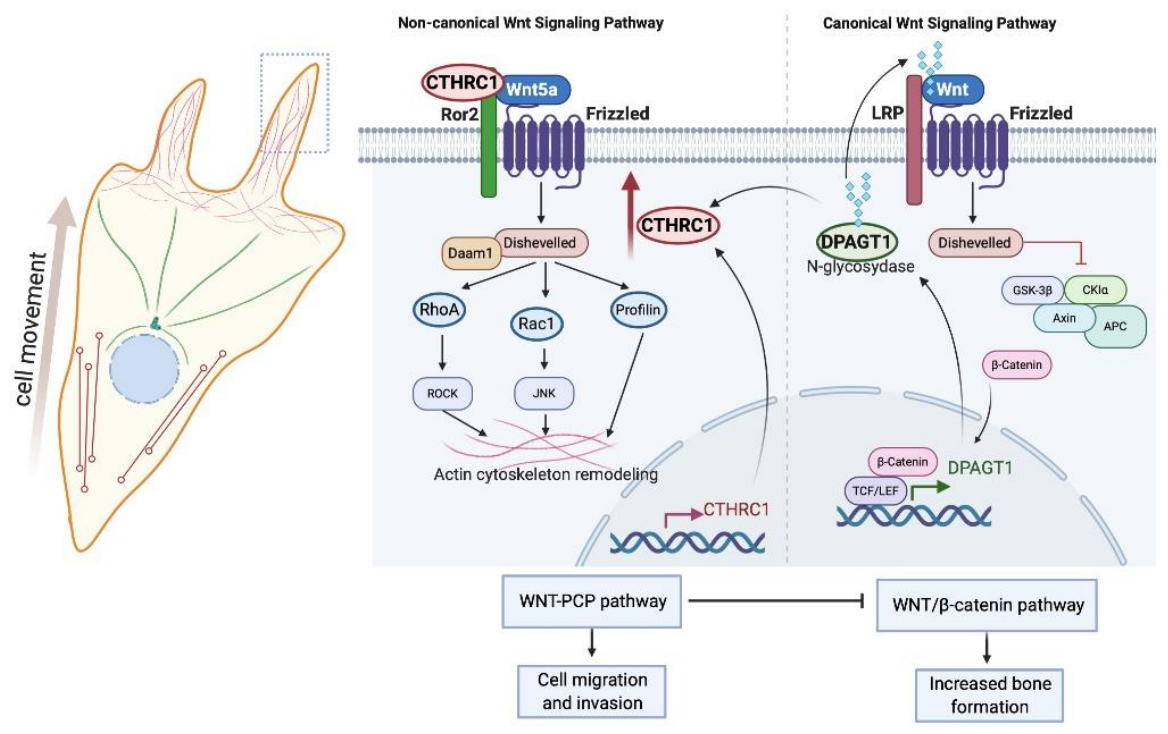

Figure 2. Role and regulation of CTHRC1 in canonical and noncanonical Wnt signaling. DPAGT1 expression is induced in response to activation of canonical Wnt/ $\beta$-catenin signaling. DPAGT1 then mediates the $\mathrm{N}$-glycosylation of CTHRC1, thereby enhancing protein stability and secretion of CTHRC1. CTHRC1, in turn, attenuates signaling by the canonical Wnt/ $\beta$-catenin pathway and induces cytoskeletal reorganization and cell movement via activation of noncanonical Wnt/PCP signaling. Secreted CTHRC1 activates the Wnt/PCP pathway by acting as a co-receptor and promoting the formation of the Wnt5A/Fzd/Ror2 complex.

Better defined is the action of CTHRC1 on the noncanonical Wnt/PCP signaling branch. Various studies have shown that CTHRC1 can activate the Wnt/PCP signalling pathway by acting as a coreceptor for formation of Wnt/Fzd/Ror2 complexes [39]. Consistent with such a role, CTHRC1 has been reported to interact with several PCP core components, including multiple Fzd (Frizzled) receptors (Fzd3, Fzd5 and Fzd6), non-canonical Wnt proteins (Wnt3A and Wnt5A), and the noncanonical co-receptor Ror2 (Receptor Tyrosine Kinase Like Orphan Receptor 2), but not with LRP6 (Low-density lipoprotein receptor-related protein 6) and the PCP component Vangl2 (VANGL planar cell polarity protein 2) [39]. CTHRC1 appears to enhance the binding of either Wnt3a or Wnt5a to Ror2 leading to enhanced Wnt3a-Ror2 and Wnt5a-Ror2 complex formation and stimulation of 
downstream signaling (Figure 2, [39]). Accordingly, CTHRC1 co-expressed with Wnt3a, Wnt5a and Dvl2 led to the activation of the Wnt/planar cell polarity (PCP) signaling pathway and enhanced the activation of both RhoA and Rac1 small GTPases indicating that CTHRC1 can modulate both cascades of the Wnt/PCP pathway [39]. Furthermore, Wnt/PCP and CTHRC1 may be part of an autocrine feedback mechanism, because CTHRC1 gene expression has been reported to be induced upon activation of Fzd6/Wnt/PCP (Figure 2; [40]). Notably, the PCP/Wnt signaling pathway regulates chondrocyte maturation and cartilage formation [41] and genetic alterations within this pathway are associated with chondrodysplasia, deregulated collagen deposition, and changes in morphological characteristics of the cartilage [41].

In addition to Fzd receptors, a recent study further identified the vertebrate-specific transmembrane protein Wnt-activated inhibitory factor 1 (Waif1, also known as trophoblast glycoprotein, TPBG; 5T4 oncofetal trophoblast glycoprotein) as a receptor for secreted CTHRC1. WntActivated Inhibitory Factor 1 (Waif1)/5T4 has been shown to inhibit Wnt/ $\beta$-catenin signaling and to concomitantly activate noncanonical Wnt pathways [42]. Taken together, these data suggest that CTHRC1 expression and function are positively regulated by the canonical Wnt signaling pathway. CTHRC1, in turn, acts as a switch between canonical and noncanonical Wnt pathways, leading to the inhibition of the canonical branch and activation of the noncanonical WNT/PCP branch of the pathway (Figure 2).

\section{CTHRC1 is associated with RA development and disease severity}

CTHRC1 was first linked to RA pathogenesis through the genetic association of Cthrc1 gene polymorphisms with attenuation of proteoglycan-induced (PGIA) and collagen antibody-induced murine arthritis (CAIA) [43-46]. Cthrc1 is located within the proteoglycan induced arthritis 8 (Pgia8) locus of mouse chromosome 15, which controls PGIA severity in a sex-specific manner [43-46]. In Pgia8-congenic male mice, expression of all genes located within the entire locus was suppressed by $30-50 \%$ and this was linked to resistance to arthritis development [44]. However, of the over 200 genes located within this locus, Cthrc1 expression exhibited the strongest correlation with arthritis severity and levels of the pro-inflammatory cytokines IL-6 and IL-1 $\beta$ [44].

Notably, while Cthrc1 was the most significantly down-regulated gene in the locus, it also exhibited marked co-expression with several other genes located within the Pgia8 locus: the Wnt signaling components R-spondyn (Rspo2) and Syndecan 2 (Sdc2), ADAM metallopeptidase with thrombospondin type 1 motif 12 (Adamts12), as well as Complement $\mathrm{C} 1 \mathrm{q}$ and tumor necrosis factor related protein 3 (C1qtnf3) [44]. All these genes were significantly repressed in male mice and linked to inflammation. Co-expression of $C t h r c 1$ with $R s p o 2$ and $S d c 2$ supports the notion that there is a link between CTHRC1 and the Wnt signaling pathway in RA. Adamts12 is genetically associated with several inflammatory conditions, including asthma, Crohn's disease, and RA $[47,48]$. In RA, Adamts 12 is one of the enzymes triggering cartilage destruction by degrading cartilage oligomeric matrix protein (COMP) [44,49]. C1qtnf3 (also named CTRP3 for "C1q/TNF-related protein-3") is an adipokine with broad immunomodulatory and metabolic functions [50]. C1qtnf3/CTRP3 is highly expressed in several mouse models of arthritis and attenuates systemic inflammation and arthritis severity, suggestive of a protective role [51,52].

Importantly, the syntenic region to the Pgias locus of mouse chromosome 15 in the human genome is also associated with RA development, serum rheumatoid factor, and efficacy of anti-TNF$\alpha$ treatment of RA patients [53,54,55,56]. The linkage of $C t h r c 1$ with arthritis development and disease severity in mice therefore raised the question whether the corresponding human locus may be similarly linked to RA development in patients. Consistent with such a notion, we recently showed that CTHRC1 protein is significantly and specifically elevated in the plasma of RA patients [7]. Importantly, CTHRC1 plasma levels were low or undetectable in healthy controls, as well as in OA and ReA patients [7]. In addition, CTHRC1 levels were positively associated with RA disease markers, such as RF, anti-citrullinated protein antibodies (ACPA), and C-reactive protein (CRP). CTHRC1 also correlated significantly with RA disease activity based on the combined index of the 28-joint disease activity score (DAS28), the combined score DAS28-CRP, and with a panel of pro- 


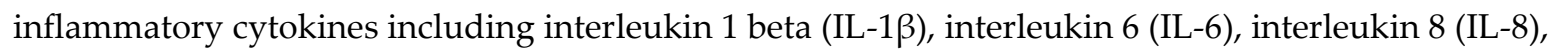
and interferon gamma (IFN $\gamma$ ) [7]. These findings corroborate observations in murine models of arthritis and indicate that CTHRC1 may have potential use as a biomarker for enhanced differential RA diagnosis.

\section{Invasive synoviocytes are major drivers of joint destruction in RA}

Synovial hyperplasia is a hallmark of RA pathogenesis and characterized by the formation of pannus. The arthritic pannus is a multicellular vascularized tissue composed of cells of both mesenchymal and hematopoietic origin (FLS; Figure 3) [34,35]. In response to synovial inflammation, pannus tissue invades cartilage and bone resulting in major damage of the intimal lining and sub lining layers of the synovial tissue and, eventually, resulting in joint destruction. Rheumatoid synoviocytes, which consist of fibroblast-like synoviocytes (FLSs) and synovial macrophages (SMs), are crucial for the progression of rheumatoid arthritis (RA) as they form the leading edge of pannus, which promotes inflammation and produces proteinases able to cause destruction of articular cartilage (Figure 3, $[57,58])$. RA-FLS may contribute to RA pathogenesis by locally producing proinflammatory cytokines and mediators of inflammation, including IL-6, IL-8, IL-15 and stromal cellderived factor 1 (SDF-1), which are essential in lymphocyte penetration into the synovium [59-63]. Activated RA-FLS in particular are key drivers of bone erosion; these cells become proliferative, migratory, and invasive in response to inflammatory signals and are a source of numerous proinflammatory cytokines, growth factors, and cartilage- and bone-degrading proteases. (Figure 3; [5963]). Importantly, RA-FLS also co-operate with macrophage-like progenitor cells leading to local formation of osteoclasts, which invade the subchondral bone using acid attack and acidic proteinases (Figure 3), thus establishing an autocrine feedback network that promotes synovial inflammation and increases FLS motility and proliferation [63]. RA synoviocytes can initiate osteoclast differentiation by RANK-RANKL (Receptor activator of nuclear factor $\kappa \mathrm{B} /$ Receptor activator of nuclear factor kappa-B ligand) interactions (Figure 3), where RANKL is expressed on FLS and T cells besides monocytes [64]. In addition, pro-inflammatory cytokines like TNF $\alpha$ and IL-1 $\beta$ protect FLS from Fasmediated cell death (Figure 3), thus preventing the elimination of RA-FLS from the inflamed synovium $[63,65]$.

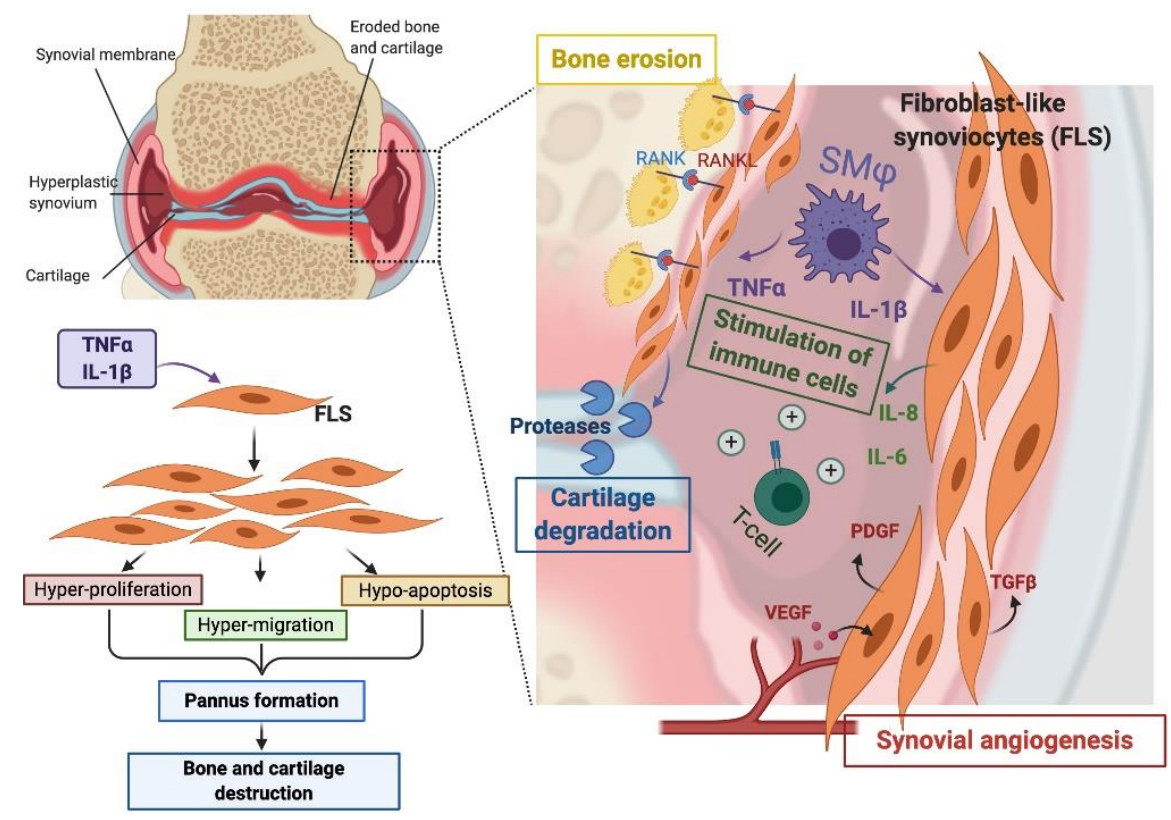

Figure 3. Role of synoviocytes in pannus formation and bone erosion in rheumatoid arthritis. Activation of fibroblast-like synoviocytes by cytokines (TNF $\alpha$ and IL-1 $\beta$ ) secreted by synovial macrophages $(\mathrm{SM} \phi)$ leads to the acquisition of a hyper-proliferative, hyper-migratory, and hypoapoptotic phenotype that contributes to the formation of a hyperplastic synovium. Activated synoviocytes in the pannus play a central role in the recruitment and stimulation of immune cells, the 
vascularization of the pannus through activation of angiogenesis, and the promotion of cartilage and bone erosion.

Several studies have shown that activated RA-FLS exhibit invasive characteristics reminiscent of cancer cells. FLS from patients with RA promote pannus angiogenesis and behave like locally invasive tumor cells that are capable of spreading the disease to as yet unaffected joints via vasculature-independent routes [66]. The invasive properties of RA-FLS from RA patients as well as from murine arthritis models directly correlate with the rate of joint destruction and deformities, and poor disease outcome $[67,68]$. The mechanisms of the enhanced aggressiveness and motility of RA-FLS and their cellular differentiation pathways have not been adequately studied. Somatic mutations in the p53 tumor suppressor gene and activated expression of ras, c-myc, c-fos, and raf oncogenes contribute to the pathological FLS phenotype in arthritic synovium [63]. FLS also express many tumor-specific markers, including podoplanin, a pro-migratory glycoprotein [69], and cadherin 11 (CDH11), which is an important marker of pannus formation and acquisition of the invasive phenotype by activated FLS [63,70].

\section{RA-FLS are one source of CTHRC1}

Significantly, the immunohistochemical staining of CAIA mouse synovium showed that CTHRC1 protein levels were highly elevated in pannus compared to normal synovial tissue and concentrated in fibroblast-like synoviocytes (FLS) located at the forefront of the invasive front attacking the bone and cartilage [71]. The expression in RA-FLS may reflect a role of CTHRC1 in synoviocyte migration [71] and invasion and, thus, the pathological changes leading to joint and bone erosion. Such a notion would be consistent with a recent study showing that CTHRC1 is expressed in two different FLS subpopulations in the synovium that are characterized by the protein levels of CD34, THY1, and cadherin 11 (CDH11): a CD34+ THY- CDH11+ fibroblast subtype, which is implicated in monocyte recruitment in the synovium through secretion of IL-6, CXCL12, and CCL2, and a CD34- $\mathrm{THY}^{+}{ }^{+} \mathrm{CDH} 11^{+}$subtype, which is migratory, invasive, promotes osteoclastogenesis, and is significantly expanded in RA versus OA and associated with pathological changes [8]. Overall, these studies point to a subpopulation of activated synoviocytes of arthritic pannus with tumor-like properties as one potential source of CTHRC1 detected in the plasma of RA patients.

At present, we can only speculate about the mechanisms governing CTHRC1 expression in RAFLS and is function is in the synovium. Among many other signaling pathways, canonical and noncanonical branches of the Wnt signaling pathway are considered to play major roles in RA pathogenesis in part by modulating the activation of FLS and by promoting the production of proinflammatory cytokines and chemokines. In vitro studies showed upregulated expression of $\beta$-catenin in FLS obtained from RA patients (RA-FLS) in response to activation of the canonical Wnt signaling pathway, which eventually leads to stable activation of RA-FLS [72]. In addition, activation of the noncanonical branch of the Wnt pathway in RA-FLS may contribute to the pro-inflammatory microenvironment of the pannus and promote the local recruitment of immune cells. Importantly, non-canonical PCP-Wnt pathway members are overexpressed in the RA synovium [72]. Kim et al. demonstrated that Wnt5a is the main precursor of cytokine production during inflammation [73]. Wnt5a expression was observed in RA-FLS, but not in normal tissue [74], and Wnt5a expression in RA-FLS led to enhanced production of pro-inflammatory cytokines and chemokines $[73,75]$. These data suggest that Wnt5a mediated signaling leads to upregulated production of cytokines and chemokines and causes recruitment of leucocytes into the synovium to enhance RA disease progression. Blockade of non-canonical Wnt5a/Fzd5 signaling leads to downregulation of IL-6, IL-15 and RANKL, which inhibits RA-FLS activation [72,76]. In addition, the Wnt5a/Ror2 complex also plays a crucial role in bone marrow derived mesenchymal stem cell differentiation into osteoblasts [73] suggesting that activation of non-canonical Wnt signaling in the arthritic synovium might affect bone homeostasis by modulating osteoblastogenesis.

A potential role of CTHRC1 as part of canonical and noncanonical Wnt signaling pathways in RA-FLS has not yet been established. However, given that CTHRC1 is known to stabilize the 
Wnt/Frizzled complex and given its pro-migratory function [40], CTHRC1 could play a critical role in Wnt/PCP signaling in the synovium, thus promoting inflammatory cell migration and cell differentiation. Characterization of the functional roles of the unique fibroblast subsets of FLS marked by CTHRC1-expression and evaluation of the functional significance of CTHRC1 expression in these FLS should provide novel insights into CTHRC1's role in RA pathogenesis.

\section{CTHRC1 plays a central role in bone remodeling}

Bone erosion is a central aspect of RA and is associated with disease severity [61]. Bone lesions both within and around the affected joints often appear early in the disease and can be accompanied by widespread osteoporosis in some patients without effective treatment. These lesions are the result of deregulated bone homeostasis due to enhanced osteoclast differentiation associated with bone resorption and the inhibition of osteoblast-mediated bone formation [61]. Osteoclasts are multinucleated cells that differentiate from monocyte/macrophage precursors under osteoblast/osteocyte control [61]. Osteoblasts regulate osteoclast differentiation and function via colony stimulating factor-1 (CSF-1) and RANKL [61]. RANKL, which binds to its receptor RANK expressed on the surface of osteoclast precursors [77], is also secreted by osteocytes. Furthermore, osteoblasts secrete osteoprotegerin (OPG), a soluble receptor for RANKL, which inhibits osteoclastogenesis by blocking the RANKL-RANK interaction, and WNT5A, which contributes to the regulation of osteoclastogenesis through activation of noncanonical Wnt signaling [78]. In addition to RANKL, osteocytes produce sclerostin (SOST), a secreted protein that attenuates osteoblast differentiation and promotes osteoclastogenesis by inhibiting the Wnt/ $\beta$-catenin pathway [79]. Studies showed that RANKL/RANK deficient mice develop osteopetrosis, whereas OPG deficient mice develop osteoporosis of trabecular and cortical bone [48-51].

Importantly, CTHRC1 has emerged as a key factor controlling osteoblast-osteoclast cross-talk to couple bone resorption to bone formation $[9,10,80,81]$. The regulatory role of CTHRC1 on osteoblastosteoclast crosstalk has been clearly demonstrated in vivo in mouse studies. Accordingly, loss of CTHRC1 function in mice was shown to result in decreased bone mass and decreased bone formation due to impaired coupling processes $[9,10,82]$. In contrast, overexpression of Cthrc1 increased bone mass through stimulation of bone formation in transgenic animals $[9,10,82]$.

However, the cellular source of secreted CTHRC1 and the precise role of CTHRC1 in bone biology are currently somewhat controversial. Several studies reported CTHRC1 to be secreted from osteoclasts and to stimulate osteoblast differentiation $[9,10,80,81]$. This effect appears to be mediated, at least in part, through WAIF1/5T4 expressed on stromal cells that regulates Wnt/ $\beta$-catenin signaling $[39,81]$. WAIF1/5T4 acts as a receptor for osteoclast-derived CTHRC1 likely leading to the formation of a ternary complex by CTHRC1, WAIF1, and Ror2 on the osteoblast surface, which then mediates osteoblast-osteoclast crosstalk for bone remodeling [39,81]. Consistent with the notion that Cthrc1 is produced by osteoclasts to influence bone remodeling, cell-type specific ablation of Cthrc1 expression in murine osteoblasts showed no significant change in bone mass whereas osteoclast-specific deletion of Cthrc1 lead to bone loss [10]. Based on these data, Takeshita et al concluded that Cthrc1 is secreted by osteoclasts and has a major role in bone remodeling [10].

In contrast, Jin et al. reported that Cthrc1 is produced by osteocytes and osteoblasts, but not osteoclasts and that loss of Cthrc1 expression in a Cthrc1 knockout mouse model blocked osteoclast differentiation, as well as joint destruction [82]. In particular, calvarial osteoblasts from Cthrc1 null mice exhibited reduced osteogenic differentiation when compared to wildtype mice [82]. Consistent with these findings, the authors showed that Cthrc1 is highly expressed by osteoblasts lining the trabecular and cortical bone surface of wild type mice and by some, but not all, osteocytes, whereas multinucleated osteoclasts did not express Cthrc1 [82]. Furthermore, osteoclast differentiation from bone marrow derived monocytes was normal in this $C t h r c 1$ null mouse model. The role of Cthrc1 in arthritis development was also assessed by the same authors in the collagen antibody-induced arthritis mouse model. Notably, they found that overexpression of Cthrc1 blocked osteoclast differentiation via inhibition of NFKB-dependent signaling, indicating that Cthrc1 may be part of a protective repair mechanism activated in response to inflammation in the synovium [82]. 
Overall, the molecular and cellular basis for the discrepancy between data reported from different laboratories is not clear. A major difference may the distinct detection methods used to visualize Cthrc1 expression in knockout mice. Alternatively, Cthrc1 may affect bone homeostasis through a combination of mechanisms and pathways depending on the tissue context and the presence or absence of inflammatory conditions. Additional studies in both cellular and in vivo systems will be required to resolve the discrepancy between the data reported by different investigators and to clarify the origin of Cthrc1. Regardless of this discrepancy, the role of Cthrc1 in bone remodeling has been firmly established in animal models and this may have implications for the development of cartilage and bone erosion in RA patients that can guide future studies.

\section{Sex disparity and CTHRC1}

Rheumatoid arthritis occurs more frequently in women (about $75 \%$ ) and symptoms are more pronounced in this population $[23,24]$. In particular, the influence of sex hormones on the immune response is well established [83] and estrogens are one of the causes of female predominance in RA and highly linked to disease severity and effects on bone remodeling [28]. In this regard, it is interesting to note that the murine pgia8 locus conferred sex-specific attenuation of arthritis only in male, but not female mice [28]. Furthermore, the expression of Cthrc1, metalloproteinase Adamts12, $R s p o 2$ and $S d c 2$ genes was not only highly associated with disease severity, but was also linked to the sex-specific effects conferred by the pgia8 locus on arthritis resistance [28]. The attenuation of Cthrc1 expression in male mice was linked to the pgia 8 locus because Cthrc1 mRNA levels were equal in wild type male and female mice [28]. The molecular basis for these sex-specific effects is not known. However, recent reports show that Cthrc1 also has a diverse effect on bone formation in male and female mice. Bone histomorphometry, micro-computed tomography analysis and functional readouts of bone strength showed bone formation impairment of trabecular and cortical bone in male Cthrc1 null mice with a significant reduction in bone mass, whereas female Cthrc1 null mice exhibited impairment only in trabecular bone [30]. Taken together, these results indicate that in mice the sexspecific disparities in RA are linked to transcriptional regulation of Cthrc1 and genes involved in cartilage degradation (Adamts12) and canonical and noncanonical Wnt signalling (Rspo2, Sdc2). Likewise, Cthrc1 confers sex-specific effects to bone formation in mice. Whether CTHRC1 expression or function is also linked to the sex-bias phenotype of RA in human patients remains to be shown.

\section{Conclusion}

In this review, we described recent advances examining the role of CTHRC1 in RA pathogenesis. Available data suggests that CTHRC1 represents a promising new diagnostic and potentially also prognostic biomarker of RA. The proposed role of CTHRC1 in canonical and noncanonical Wnt signaling during synovium proliferation, bone formation/resorption and linkage to disease severity will require further studies. Likewise, additional studies will be necessary to reveal the precise function of CTHRC1 in RA pathogenesis and its therapeutic proposition. Nevertheless, the similarity in CTHRC1 function in cancer cells and synoviocytes is intriguing and provides a promising basis for future therapeutic and diagnostic development of CTHRC1.

Table 1. Potential cellular sources of CTHRC1 within the joint.

\begin{tabular}{ccc}
\hline Cell type & Possible effect on cells & Reference \\
\hline $\begin{array}{c}\text { Rheumatoid arthritis } \\
\text { fibroblast-like } \\
\text { synoviocytes (RA-FLS) } \\
\text { Osteoblasts }\end{array}$ & Activation and initiation of cartilage destruction & [71] \\
Octivation of osteoclastogenesis and bone destruction \\
Osteoclasts & $\begin{array}{c}\text { Inhibition of monocyte-osteoclast differentiation and } \\
\text { osteoclast driven bone resorption, inhibition of NFkB } \\
\text { activation }\end{array}$ & {$[82]$} \\
\hline [9,10,80,81]
\end{tabular}


CTHRC1 secreted from osteocytes and osteoblasts functions as an inhibitor of osteoclast differentiation via inhibition of NFkB-dependent signaling

Author Contributions: A.-M.; conceptualization, writing-original draft preparation. L. M.; writing-figure conceptualization and preparation. S. L.; writing - original draft preparation. J. K.; conceptualization; writingoriginal draft preparation and editing; funding acquisition. All authors read and approved the final manuscript.

Funding: This work was funded by a Nazarbayev University research award (Project number 064.01.01 SST 2016022 to JK).

Conflicts of Interest: The authors declare that they have no competing interests.

\section{Abbreviations}

\begin{tabular}{|c|c|}
\hline ACPA & Anti-citrullinated protein antibody \\
\hline ADAMTs12 & A disintegrin and metallopeptidase with thrombospondin type 1 motif 12 \\
\hline Ang2 & angiopoietin-2 \\
\hline $\mathrm{BMP} 2 / 4$ & Bone morphogenetic protein $2 / 4$ \\
\hline CAIA & Collagen Antibody-Induced Arthritis \\
\hline CDH11 & cadherin 11 \\
\hline COMP & cartilage oligomeric matrix protein \\
\hline C1qtnf3 & Complement $\mathrm{C} 1 \mathrm{q}$ tumor necrosis factor-related protein 3 \\
\hline CSF-1: & Colony stimulating factor 1 \\
\hline CRP & C-reactive protein \\
\hline CTHRC1 & Collagen triple helix repeat-containing 1 protein \\
\hline DPAGT 1 & Dolichyl-Phosphate N-Acetylglucosaminephosphotransferase 1 \\
\hline Dvl & disheveled \\
\hline Fzd & Frizzled \\
\hline GSK3 $\beta$ & Glycogen synthase kinase 3beta \\
\hline IL-1/6/8/11/15 & Interleukin $1 / 6 / 8 / 11 / 15$ \\
\hline INF- $\gamma$ & Interferon gamma \\
\hline LEF & Lymphoid enhancer binding factor \\
\hline LGR $4 / 5$ & Leucine-rich repeat-containing G-protein coupled receptor $4 / 5$ \\
\hline LRP & Lipoprotein receptor-related protein \\
\hline OA & Osteoarthritis \\
\hline OPG & Osteoprotegerin \\
\hline PCP & Pathway: Planar cell polarity pathway \\
\hline RA & Rheumatoid arthritis \\
\hline RA-FLS & Rheumatoid arthritis fibroblast like synoviocyte \\
\hline RANKL & Receptor Activator of Nuclear factor-Kappa B ligand \\
\hline RF & Rheumatoid factor \\
\hline Ror2 & Receptor tyrosine kinase-like orphan receptor 2 \\
\hline RSPO2 & R-spondin 2 \\
\hline SDF1 & Stromal cell-derived factor 1 \\
\hline SMAD $2 / 3$ & $\begin{array}{l}\text { The abbreviation refers to the homologies to the Caenorhabditis elegans "small" worm } \\
\text { phenotype and Drosophila MAD ("Mothers Against Decapentaplegic") family of genes }\end{array}$ \\
\hline SOST & Sclerostin \\
\hline TPBG & trophoblast glycoprotein \\
\hline TCF & $\mathrm{T}$ cell factor \\
\hline TGF $\beta$ & Transforming growth factor beta \\
\hline TNF $\alpha$ & Tumor necrosis factor alpha \\
\hline Vang12 & VANGL planar cell polarity protein 2 \\
\hline Waif & Wnt-activated inhibitory factor 1 \\
\hline Wnt & Wingless and Int-1 \\
\hline ZNRF3 & Zinc And Ring Finger 3; RNF 43: Ring Finger Protein 43 \\
\hline
\end{tabular}

\section{References}


1. Gibofsky, A. Epidemiology, pathophysiology, and diagnosis of rheumatoid arthritis: A Synopsis. Am J Manag Care 2014, 20, S128-135.

2. Smolen, J.S.; Aletaha, D.; Barton, A.; Burmester, G.R.; Emery, P.; Firestein, G.S.; Kavanaugh, A.; McInnes, I.B.; Solomon, D.H.; Strand, V., et al. Rheumatoid arthritis. Nat Rev Dis Primers 2018, 4, 18001, doi:10.1038/nrdp.2018.1.

3. Feldmann, M. Development of anti-TNF therapy for rheumatoid arthritis. Nat Rev Immunol 2002, 2, 364371, doi:10.1038/nri802.

4. Rein, P.; Mueller, R.B. Treatment with Biologicals in Rheumatoid Arthritis: An Overview. Rheumatol Ther 2017, 4, 247-261, doi:10.1007/s40744-017-0073-3.

5. Brzustewicz, E.; Bryl, E. The role of cytokines in the pathogenesis of rheumatoid arthritis--Practical and potential application of cytokines as biomarkers and targets of personalized therapy. Cytokine 2015, 76, 527536, doi:10.1016/j.cyto.2015.08.260.

6. van Delft, M.A.M.; Huizinga, T.W.J. An overview of autoantibodies in rheumatoid arthritis. J Autoimmun 2020, 110, 102392, doi:10.1016/j.jaut.2019.102392.

7. Myngbay, A.; Bexeitov, Y.; Adilbayeva, A.; Assylbekov, Z.; Yevstratenko, B.P.; Aitzhanova, R.M.; Matkarimov, B.; Adarichev, V.A.; Kunz, J. CTHRC1: A New Candidate Biomarker for Improved Rheumatoid Arthritis Diagnosis. Front Immunol 2019, 10, 1353, doi:10.3389/fimmu.2019.01353.

8. Mizoguchi, F.; Slowikowski, K.; Wei, K.; Marshall, J.L.; Rao, D.A.; Chang, S.K.; Nguyen, H.N.; Noss, E.H.; Turner, J.D.; Earp, B.E., et al. Functionally distinct disease-associated fibroblast subsets in rheumatoid arthritis. Nat Commun 2018, 9, 789, doi:10.1038/s41467-018-02892-y.

9. Kimura, H.; Kwan, K.M.; Zhang, Z.; Deng, J.M.; Darnay, B.G.; Behringer, R.R.; Nakamura, T.; de Crombrugghe, B.; Akiyama, H. Cthrc1 is a positive regulator of osteoblastic bone formation. PLoS One 2008, 3, e3174, doi:10.1371/journal.pone.0003174.

10. Takeshita, S.; Fumoto, T.; Matsuoka, K.; Park, K.A.; Aburatani, H.; Kato, S.; Ito, M.; Ikeda, K. Osteoclastsecreted CTHRC1 in the coupling of bone resorption to formation. J Clin Invest 2013, 123, 3914-3924, doi:10.1172/JCI69493.

11. Pyagay, P.; Heroult, M.; Wang, Q.; Lehnert, W.; Belden, J.; Liaw, L.; Friesel, R.E.; Lindner, V. Collagen triple helix repeat containing 1, a novel secreted protein in injured and diseased arteries, inhibits collagen expression and promotes cell migration. Circ Res 2005, 96, 261-268, doi:10.1161/01.RES.0000154262.07264.12.

12. Leclere, L.; Nir, T.S.; Bazarsky, M.; Braitbard, M.; Schneidman-Duhovny, D.; Gat, U. Dynamic Evolution of the Cthrc1 Genes, a Newly Defined Collagen-Like Family. Genome Biol Evol 2020, 12, 3957-3970, doi:10.1093/gbe/evaa020.

13. Li, J.; Cao, J.; Li, M.; Yu, Y.; Yang, Y.; Xiao, X.; Wu, Z.; Wang, L.; Tu, Y.; Chen, H. Collagen triple helix repeat containing-1 inhibits transforming growth factor-b1-induced collagen type I expression in keloid. $\mathrm{Br} \mathrm{J}$ Dermatol 2011, 164, 1030-1036, doi:10.1111/j.1365-2133.2011.10215.x.

14. Zhang, R.; Cao, Y.; Bai, L.; Zhu, C.; Li, R.; He, H.; Liu, Y.; Wu, K.; Liu, F.; Wu, J. The collagen triple helix repeat containing 1 facilitates hepatitis $\mathrm{B}$ virus-associated hepatocellular carcinoma progression by regulating multiple cellular factors and signal cascades. Mol Carcinog 2015, 54, 1554-1566, doi:10.1002/mc.22229.

15. Bai, L.; Zhang, W.; Tan, L.; Yang, H.; Ge, M.; Zhu, C.; Zhang, R.; Cao, Y.; Chen, J.; Luo, Z., et al. Hepatitis B virus hijacks CTHRC1 to evade host immunity and maintain replication. J Mol Cell Biol 2015, 7, 543-556, doi:10.1093/jmcb/mjv048.

16. Bian, Z.; Miao, Q.; Zhong, W.; Zhang, H.; Wang, Q.; Peng, Y.; Chen, X.; Guo, C.; Shen, L.; Yang, F., et al. Treatment of cholestatic fibrosis by altering gene expression of Cthrc1: Implications for autoimmune and non-autoimmune liver disease. J Autoimmun 2015, 63, 76-87, doi:10.1016/j.jaut.2015.07.010.

17. LeClair, R.; Lindner, V. The role of collagen triple helix repeat containing 1 in injured arteries, collagen expression, and transforming growth factor beta signaling. Trends Cardiovasc Med 2007, 17, 202-205, doi:10.1016/j.tcm.2007.05.004.

18. Bauer, Y.; Tedrow, J.; de Bernard, S.; Birker-Robaczewska, M.; Gibson, K.F.; Guardela, B.J.; Hess, P.; Klenk, A.; Lindell, K.O.; Poirey, S., et al. A novel genomic signature with translational significance for human idiopathic pulmonary fibrosis. Am J Respir Cell Mol Biol 2015, 52, 217-231, doi:10.1165/rcmb.2013-0310OC.

19. Binks, A.P.; Beyer, M.; Miller, R.; LeClair, R.J. Cthrc1 lowers pulmonary collagen associated with bleomycin-induced fibrosis and protects lung function. Physiol Rep 2017, 5, doi:10.14814/phy2.13115. 
20. Li, J.; Wang, Y.; Ma, M.; Jiang, S.; Zhang, X.; Zhang, Y.; Yang, X.; Xu, C.; Tian, G.; Li, Q., et al. Autocrine CTHRC1 activates hepatic stellate cells and promotes liver fibrosis by activating TGF-beta signaling. EBioMedicine 2019, 40, 43-55, doi:10.1016/j.ebiom.2019.01.009.

21. Tsukui, T.; Sun, K.H.; Wetter, J.B.; Wilson-Kanamori, J.R.; Hazelwood, L.A.; Henderson, N.C.; Adams, T.S.; Schupp, J.C.; Poli, S.D.; Rosas, I.O., et al. Collagen-producing lung cell atlas identifies multiple subsets with distinct localization and relevance to fibrosis. Nat Commun 2020, 11, 1920, doi:10.1038/s41467-020-15647-5.

22. Li, Y.K.; Li, Y.M.; Li, Y.; Wei, Y.R.; Zhang, J.; Li, B.; You, Z.R.; Chen, Y.; Huang, B.Y.; Miao, Q., et al. CTHRC1 expression in primary biliary cholangitis. J Dig Dis 2019, 20, 371-376, doi:10.1111/1751-2980.12791.

23. Jiang, N.; Cui, Y.; Liu, J.; Zhu, X.; Wu, H.; Yang, Z.; Ke, Z. Multidimensional Roles of Collagen Triple Helix Repeat Containing 1 (CTHRC1) in Malignant Cancers. J Cancer 2016, 7, 2213-2220, doi:10.7150/jca.16539.

24. Liu, J.; Li, W.; Liu, S.; Zheng, X.; Shi, L.; Zhang, W.; Yang, H. Knockdown of Collagen Triple Helix Repeat Containing 1 (CTHRC1) Inhibits Epithelial-Mesenchymal Transition and Cellular Migration in Glioblastoma Cells. Oncol Res 2017, 25, 225-232, doi:10.3727/096504016X14732772150587.

25. Jin, X.F.; Li, H.; Zong, S.; Li, H.Y. Knockdown of Collagen Triple Helix Repeat Containing-1 Inhibits the Proliferation and Epithelial-to-Mesenchymal Transition in Renal Cell Carcinoma Cells. Oncol Res 2016, 24, 477-485, doi:10.3727/096504016X14685034103716.

26. Ni, S.; Ren, F.; Xu, M.; Tan, C.; Weng, W.; Huang, Z.; Sheng, W.; Huang, D. CTHRC1 overexpression predicts poor survival and enhances epithelial-mesenchymal transition in colorectal cancer. Cancer Med 2018, 7, 5643-5654, doi:10.1002/cam4.1807.

27. Wang, Y.; Lee, M.; Yu, G.; Lee, H.; Han, X.; Kim, D. CTHRC1 activates pro-tumorigenic signaling pathways in hepatocellular carcinoma. Oncotarget 2017, 8, 105238-105250, doi:10.18632/oncotarget.22164.

28. Lee, J.; Song, J.; Kwon, E.S.; Jo, S.; Kang, M.K.; Kim, Y.J.; Hwang, Y.; Bae, H.; Kang, T.H.; Chang, S., et al. CTHRC1 promotes angiogenesis by recruiting Tie2-expressing monocytes to pancreatic tumors. Exp Mol Med 2016, 48, e261, doi:10.1038/emm.2016.87.

29. Fu, S.W.; Chen, H.Y.; Lin, X.L.; Yang, L.; Ge, Z.Z. Collagen triple helix repeat containing 1 promotes tumor angiogenesis in gastrointestinal stromal tumors. Oncol Lett 2017, 14, 7499-7505, doi:10.3892/ol.2017.7111.

30. Durmus, T.; LeClair, R.J.; Park, K.S.; Terzic, A.; Yoon, J.K.; Lindner, V. Expression analysis of the novel gene collagen triple helix repeat containing-1 (Cthrc1). Gene Expr Patterns 2006, 6, 935-940, doi:10.1016/j.modgep.2006.03.008.

31. Leclair, R.J.; Wang, Q.; Benson, M.A.; Prudovsky, I.; Lindner, V. Intracellular localization of Cthrc1 characterizes differentiated smooth muscle. Arterioscler Thromb Vasc Biol 2008, 28, 1332-1338, doi:10.1161/ATVBAHA.108.166579.

32. Duarte, C.W.; Stohn, J.P.; Wang, Q.; Emery, I.F.; Prueser, A.; Lindner, V. Elevated plasma levels of the pituitary hormone $\mathrm{Cthrc1}$ in individuals with red hair but not in patients with solid tumors. PLoS One 2014, 9, e100449, doi:10.1371/journal.pone.0100449.

33. LeClair, R.J.; Durmus, T.; Wang, Q.; Pyagay, P.; Terzic, A.; Lindner, V. Cthrc1 is a novel inhibitor of transforming growth factor-beta signaling and neointimal lesion formation. Circ Res 2007, 100, 826-833, doi:10.1161/01.RES.0000260806.99307.72.

34. Wang, P.; Wang, Y.C.; Chen, X.Y.; Shen, Z.Y.; Cao, H.; Zhang, Y.J.; Yu, J.; Zhu, J.D.; Lu, Y.Y.; Fang, J.Y. CTHRC1 is upregulated by promoter demethylation and transforming growth factor-beta1 and may be associated with metastasis in human gastric cancer. Cancer Sci 2012, 103, 1327-1333, doi:10.1111/j.13497006.2012.02292.x.

35. Kikuchi, K.; Kubo, M.; Sato, S.; Fujimoto, M.; Tamaki, K. Serum tissue inhibitor of metalloproteinases in patients with systemic sclerosis. J Am Acad Dermatol 1995, 33, 973-978, doi:10.1016/0190-9622(95)90289-9.

36. Clevers, H.; Nusse, R. Wnt/beta-catenin signaling and disease. Cell 2012, 149, 1192-1205, doi:10.1016/j.cell.2012.05.012.

37. Liu, G.; Sengupta, P.K.; Jamal, B.; Yang, H.Y.; Bouchie, M.P.; Lindner, V.; Varelas, X.; Kukuruzinska, M.A. $\mathrm{N}$-glycosylation induces the CTHRC1 protein and drives oral cancer cell migration. J Biol Chem 2013, 288, 20217-20227, doi:10.1074/jbc.M113.473785.

38. Sengupta, P.K.; Bouchie, M.P.; Kukuruzinska, M.A. N-glycosylation gene DPAGT1 is a target of the Wnt/beta-catenin signaling pathway. J Biol Chem 2010, 285, 31164-31173, doi:10.1074/jbc.M110.149195.

39. Yamamoto, S.; Nishimura, O.; Misaki, K.; Nishita, M.; Minami, Y.; Yonemura, S.; Tarui, H.; Sasaki, H. Cthrc1 selectively activates the planar cell polarity pathway of Wnt signaling by stabilizing the Wntreceptor complex. Dev Cell 2008, 15, 23-36, doi:10.1016/j.devcel.2008.05.007. 
40. Dong, B.; Vold, S.; Olvera-Jaramillo, C.; Chang, H. Functional redundancy of frizzled 3 and frizzled 6 in planar cell polarity control of mouse hair follicles. Development 2018, 145, doi:10.1242/dev.168468.

41. Wang, Y.; Li, Y.P.; Paulson, C.; Shao, J.Z.; Zhang, X.; Wu, M.; Chen, W. Wnt and the Wnt signaling pathway in bone development and disease. Front Biosci (Landmark Ed) 2014, 19, 379-407, doi:10.2741/4214.

42. Kagermeier-Schenk, B.; Wehner, D.; Ozhan-Kizil, G.; Yamamoto, H.; Li, J.; Kirchner, K.; Hoffmann, C.; Stern, P.; Kikuchi, A.; Schambony, A., et al. Waif1/5T4 inhibits Wnt/beta-catenin signaling and activates noncanonical Wnt pathways by modifying LRP6 subcellular localization. Dev Cell 2011, 21, 1129-1143, doi:10.1016/j.devcel.2011.10.015.

43. Adarichev, V.A.; Nesterovitch, A.B.; Bardos, T.; Biesczat, D.; Chandrasekaran, R.; Vermes, C.; Mikecz, K.; Finnegan, A.; Glant, T.T. Sex effect on clinical and immunologic quantitative trait loci in a murine model of rheumatoid arthritis. Arthritis Rheum 2003, 48, 1708-1720, doi:10.1002/art.11016.

44. Kudryavtseva, E.; Forde, T.S.; Pucker, A.D.; Adarichev, V.A. Wnt signaling genes of murine chromosome 15 are involved in sex-affected pathways of inflammatory arthritis. Arthritis Rheum 2012, 64, 1057-1068, doi:10.1002/art.33414.

45. Adarichev, V.A.; Vegvari, A.; Szabo, Z.; Kis-Toth, K.; Mikecz, K.; Glant, T.T. Congenic strains displaying similar clinical phenotype of arthritis represent different immunologic models of inflammation. Genes Immun 2008, 9, 591-601, doi:10.1038/gene.2008.54.

46. Glant, T.T.; Szanto, S.; Vegvari, A.; Szabo, Z.; Kis-Toth, K.; Mikecz, K.; Adarichev, V.A. Two loci on chromosome 15 control experimentally induced arthritis through the differential regulation of IL-6 and lymphocyte proliferation. J Immunol 2008, 181, 1307-1314.

47. Libioulle, C.; Louis, E.; Hansoul, S.; Sandor, C.; Farnir, F.; Franchimont, D.; Vermeire, S.; Dewit, O.; de Vos, M.; Dixon, A., et al. Novel Crohn disease locus identified by genome-wide association maps to a gene desert on 5p13.1 and modulates expression of PTGER4. PLoS Genet 2007, 3, e58, doi:10.1371/journal.pgen.0030058.

48. Kurz, T.; Hoffjan, S.; Hayes, M.G.; Schneider, D.; Nicolae, R.; Heinzmann, A.; Jerkic, S.P.; Parry, R.; Cox, N.J.; Deichmann, K.A., et al. Fine mapping and positional candidate studies on chromosome 5p13 identify multiple asthma susceptibility loci. J Allergy Clin Immunol 2006, 118, 396-402, doi:10.1016/j.jaci.2006.04.036.

49. Liu, C.J.; Kong, W.; Xu, K.; Luan, Y.; Ilalov, K.; Sehgal, B.; Yu, S.; Howell, R.D.; Di Cesare, P.E. ADAMTS12 associates with and degrades cartilage oligomeric matrix protein. J Biol Chem 2006, 281, 15800-15808, doi:10.1074/jbc.M513433200.

50. Li, Y.; Wright, G.L.; Peterson, J.M. C1q/TNF-Related Protein 3 (CTRP3) Function and Regulation. Compr Physiol 2017, 7, 863-878, doi:10.1002/cphy.c160044.

51. Kim, J.Y.; Min, J.Y.; Baek, J.M.; Ahn, S.J.; Jun, H.Y.; Yoon, K.H.; Choi, M.K.; Lee, M.S.; Oh, J. CTRP3 acts as a negative regulator of osteoclastogenesis through AMPK-c-Fos-NFATc1 signaling in vitro and RANKLinduced calvarial bone destruction in vivo. Bone 2015, 79, 242-251, doi:10.1016/j.bone.2015.06.011.

52. Murayama, M.A.; Kakuta, S.; Maruhashi, T.; Shimizu, K.; Seno, A.; Kubo, S.; Sato, N.; Saijo, S.; Hattori, M.; Iwakura, Y. CTRP3 plays an important role in the development of collagen-induced arthritis in mice. Biochem Biophys Res Commun 2014, 443, 42-48, doi:10.1016/j.bbrc.2013.11.040.

53. Wei, Z.; Li, M. Genome-wide linkage and association analysis of rheumatoid arthritis in a Canadian population. BMC Proc 2007, 1 Suppl 1, S19, doi:10.1186/1753-6561-1-s1-s19.

54. Jawaheer, D.; Seldin, M.F.; Amos, C.I.; Chen, W.V.; Shigeta, R.; Etzel, C.; Damle, A.; Xiao, X.; Chen, D.; Lum, R.F., et al. Screening the genome for rheumatoid arthritis susceptibility genes: a replication study and combined analysis of 512 multicase families. Arthritis Rheum 2003, 48, 906-916, doi:10.1002/art.10989.

55. Mukhopadhyay, N.; Halder, I.; Bhattacharjee, S.; Weeks, D.E. Two-dimensional linkage analyses of rheumatoid arthritis. BMC Proc 2007, 1 Suppl 1, S68, doi:10.1186/1753-6561-1-s1-s68.

56. Plant, D.; Bowes, J.; Potter, C.; Hyrich, K.L.; Morgan, A.W.; Wilson, A.G.; Isaacs, J.D.; Wellcome Trust Case Control, C.; British Society for Rheumatology Biologics, R.; Barton, A. Genome-wide association study of genetic predictors of anti-tumor necrosis factor treatment efficacy in rheumatoid arthritis identifies associations with polymorphisms at seven loci. Arthritis Rheum 2011, 63, 645-653, doi:10.1002/art.30130.

57. Lindqvist, E.; Jonsson, K.; Saxne, T.; Eberhardt, K. Course of radiographic damage over 10 years in a cohort with early rheumatoid arthritis. Ann Rheum Dis 2003, 62, 611-616, doi:10.1136/ard.62.7.611.

58. Machold, K.P.; Stamm, T.A.; Nell, V.P.; Pflugbeil, S.; Aletaha, D.; Steiner, G.; Uffmann, M.; Smolen, J.S. Very recent onset rheumatoid arthritis: clinical and serological patient characteristics associated with radiographic progression over the first years of disease. Rheumatology (Oxford) 2007, 46, 342-349, doi:10.1093/rheumatology/kel237. 
59. Zvaifler, N.J.; Firestein, G.S. Pannus and pannocytes. Alternative models of joint destruction in rheumatoid arthritis. Arthritis Rheum 1994, 37, 783-789, doi:10.1002/art.1780370601.

60. Gravallese, E.M. Bone destruction in arthritis. Ann Rheum Dis 2002, 61 Suppl 2, ii84-86, doi:10.1136/ard.61.suppl_2.ii84.

61. Schett, G.; Gravallese, E. Bone erosion in rheumatoid arthritis: mechanisms, diagnosis and treatment. Nat Rev Rheumatol 2012, 8, 656-664, doi:10.1038/nrrheum.2012.153.

62. Goldring, S.R.; Gravallese, E.M. Pathogenesis of bone lesions in rheumatoid arthritis. Curr Rheumatol Rep 2002, 4, 226-231, doi:10.1007/s11926-002-0069-y.

63. Bartok, B.; Firestein, G.S. Fibroblast-like synoviocytes: key effector cells in rheumatoid arthritis. Immunol Rev 2010, 233, 233-255, doi:10.1111/j.0105-2896.2009.00859.x.

64. Geusens, P. The role of RANK ligand/osteoprotegerin in rheumatoid arthritis. Ther Adv Musculoskelet Dis 2012, 4, 225-233, doi:10.1177/1759720X12438080.

65. Ohshima, S.; Mima, T.; Sasai, M.; Nishioka, K.; Shimizu, M.; Murata, N.; Yoshikawa, H.; Nakanishi, K.; Suemura, M.; McCloskey, R.V., et al. Tumour necrosis factor alpha (TNF-alpha) interferes with Fasmediated apoptotic cell death on rheumatoid arthritis (RA) synovial cells: a possible mechanism of rheumatoid synovial hyperplasia and a clinical benefit of anti-TNF-alpha therapy for RA. Cytokine 2000, 12, 281-288, doi:10.1006/cyto.1999.0552.

66. Lefevre, S.; Knedla, A.; Tennie, C.; Kampmann, A.; Wunrau, C.; Dinser, R.; Korb, A.; Schnaker, E.M.; Tarner, I.H.; Robbins, P.D., et al. Synovial fibroblasts spread rheumatoid arthritis to unaffected joints. Nat Med 2009, 15, 1414-1420, doi:10.1038/nm.2050.

67. Tolboom, T.C.; Pieterman, E.; van der Laan, W.H.; Toes, R.E.; Huidekoper, A.L.; Nelissen, R.G.; Breedveld, F.C.; Huizinga, T.W. Invasive properties of fibroblast-like synoviocytes: correlation with growth characteristics and expression of MMP-1, MMP-3, and MMP-10. Ann Rheum Dis 2002, 61, 975-980, doi:10.1136/ard.61.11.975.

68. Tolboom, T.C.; van der Helm-Van Mil, A.H.; Nelissen, R.G.; Breedveld, F.C.; Toes, R.E.; Huizinga, T.W. Invasiveness of fibroblast-like synoviocytes is an individual patient characteristic associated with the rate of joint destruction in patients with rheumatoid arthritis. Arthritis Rheum 2005, 52, 1999-2002, doi:10.1002/art.21118.

69. Ekwall, A.K.; Eisler, T.; Anderberg, C.; Jin, C.; Karlsson, N.; Brisslert, M.; Bokarewa, M.I. The tumourassociated glycoprotein podoplanin is expressed in fibroblast-like synoviocytes of the hyperplastic synovial lining layer in rheumatoid arthritis. Arthritis Res Ther 2011, 13, R40, doi:10.1186/ar3274.

70. Lee, D.M.; Kiener, H.P.; Agarwal, S.K.; Noss, E.H.; Watts, G.F.; Chisaka, O.; Takeichi, M.; Brenner, M.B. Cadherin-11 in synovial lining formation and pathology in arthritis. Science 2007, 315, 1006-1010, doi:10.1126/science.1137306.

71. Shekhani, M.T.; Forde, T.S.; Adilbayeva, A.; Ramez, M.; Myngbay, A.; Bexeitov, Y.; Lindner, V.; Adarichev, V.A. Collagen triple helix repeat containing 1 is a new promigratory marker of arthritic pannus. Arthritis Res Ther 2016, 18, 171, doi:10.1186/s13075-016-1067-1.

72. Miao, C.G.; Yang, Y.Y.; He, X.; Li, X.F.; Huang, C.; Huang, Y.; Zhang, L.; Lv, X.W.; Jin, Y.; Li, J. Wnt signaling pathway in rheumatoid arthritis, with special emphasis on the different roles in synovial inflammation and bone remodeling. Cell Signal 2013, 25, 2069-2078, doi:10.1016/j.cellsig.2013.04.002.

73. Kim, J.; Kim, J.; Kim, D.W.; Ha, Y.; Ihm, M.H.; Kim, H.; Song, K.; Lee, I. Wnt5a induces endothelial inflammation via beta-catenin-independent signaling. J Immunol 2010, 185, 1274-1282, doi:10.4049/jimmunol.1000181.

74. Rauner, M.; Stein, N.; Winzer, M.; Goettsch, C.; Zwerina, J.; Schett, G.; Distler, J.H.; Albers, J.; Schulze, J.; Schinke, T., et al. WNT5A is induced by inflammatory mediators in bone marrow stromal cells and regulates cytokine and chemokine production. J Bone Miner Res 2012, 27, 575-585, doi:10.1002/jbmr.1488.

75. Sen, M.; Lauterbach, K.; El-Gabalawy, H.; Firestein, G.S.; Corr, M.; Carson, D.A. Expression and function of wingless and frizzled homologs in rheumatoid arthritis. Proc Natl Acad Sci U S A 2000, 97, 2791-2796, doi:10.1073/pnas.050574297.

76. Sen, M.; Chamorro, M.; Reifert, J.; Corr, M.; Carson, D.A. Blockade of Wnt-5A/frizzled 5 signaling inhibits rheumatoid synoviocyte activation. Arthritis Rheum 2001, 44, 772-781, doi:10.1002/15290131(200104)44:4<772::AID-ANR133>3.0.CO;2-L. 
77. Kong, Y.Y.; Yoshida, H.; Sarosi, I.; Tan, H.L.; Timms, E.; Capparelli, C.; Morony, S.; Oliveira-dos-Santos, A.J.; Van, G.; Itie, A., et al. OPGL is a key regulator of osteoclastogenesis, lymphocyte development and lymph-node organogenesis. Nature 1999, 397, 315-323, doi:10.1038/16852.

78. Tanaka, Y.; Ohira, T. Mechanisms and therapeutic targets for bone damage in rheumatoid arthritis, in particular the RANK-RANKL system. Curr Opin Pharmacol 2018, 40, 110-119, doi:10.1016/j.coph.2018.03.006.

79. Delgado-Calle, J.; Sato, A.Y.; Bellido, T. Role and mechanism of action of sclerostin in bone. Bone 2017, 96, 29-37, doi:10.1016/j.bone.2016.10.007.

80. Wang, C.; Gu, W.; Sun, B.; Zhang, Y.; Ji, Y.; Xu, X.; Wen, Y. CTHRC1 promotes osteogenic differentiation of periodontal ligament stem cells by regulating TAZ. J Mol Histol 2017, 48, 311-319, doi:10.1007/s10735017-9729-0.

81. Matsuoka, K.; Kohara, Y.; Naoe, Y.; Watanabe, A.; Ito, M.; Ikeda, K.; Takeshita, S. WAIF1 Is a Cell-Surface CTHRC1 Binding Protein Coupling Bone Resorption and Formation. J Bone Miner Res 2018, 10.1002/jbmr.3436, doi:10.1002/jbmr.3436.

82. Jin, Y.R.; Stohn, J.P.; Wang, Q.; Nagano, K.; Baron, R.; Bouxsein, M.L.; Rosen, C.J.; Adarichev, V.A.; Lindner, V. Inhibition of osteoclast differentiation and collagen antibody-induced arthritis by CTHRC1. Bone 2017, 97, 153-167, doi:10.1016/j.bone.2017.01.022.

83. Taneja, V. Sex Hormones Determine Immune Response. Front Immunol 2018, 9, 1931, doi:10.3389/fimmu.2018.01931. 